\title{
À la recherche des institutions optimales du marché du travail
}

Peter Auer

\section{OpenEdition}

\section{Journals}

Édition électronique

URL : http://journals.openedition.org/travailemploi/4075

DOI : 10.4000/travailemploi.4075

ISSN : 1775-416X

Éditeur

DARES - Ministère du Travail

\section{Édition imprimée}

Date de publication : 30 mars 2009

Pagination : 7-23

ISSN : 0224-4365

\section{Référence électronique}

Peter Auer, «À la recherche des institutions optimales du marché du travail », Travail et Emploi [En

ligne], 117 | janvier-mars 2009, mis en ligne le 30 mars 2011, consulté le 10 décembre 2020. URL: http://journals.openedition.org/travailemploi/4075; DOI : https://doi.org/10.4000/travailemploi.4075

(C) Direction de l'animation de la recherche, des études et des statistiques (Dares) 


\title{
À la recherche des institutions optimales du marché du travail
}

\author{
Peter Auer (*)
}

Cet article se penche sur les débats qui entourent la recherche d'institutions optimales de régulation du marché du travail européen, renforcée par le contexte actuel de crise financière et économique. Différentes théories et courants s'opposent sur l'impact positif ou négatif des institutions sur le fonctionnement du marché du travail. L'article analyse ces profondes divergences d'opinions sur l'efficacité de la législation de la protection de l'emploi, du système des prestations d'assurancechômage, des programmes de politique active de l'emploi, souvent incriminés de fausser le libre jeu du marché du travail, et questionne l'efficacité des politiques actives du marché du travail. Il discute ensuite de l'option que représente la flexicurité et de ses conditions de mise en auvre, et pose la question de savoir si elle survivra au test de la crise actuelle.

L'effet de la mondialisation sur l'emploi a provoqué un nouvel intérêt pour les institutions du marché du travail, particulièrement quant à leur capacité de permettre l'ajustement flexible des entreprises aux changements structurels et conjoncturels intensifiés par l'ouverture des marchés mondiaux sans mettre en péril la sécurité des travailleurs. $\mathrm{La}$ crise actuelle, déclenchée sur les marchés financiers mondiaux (éléments à la fois de renforcement et de déstabilisation de la mondialisation), a atteint l'économie réelle et renouvelle donc la question de la performance des différentes institutions du marché du travail à le gérer. Bien que cet article ait été écrit en grande partie avant qu'elle ne survienne, les débats sur les institutions «optimales» du marché de travail sont aujourd'hui d'une grande opportunité. Même si ce n'est qu'après la crise que l'on saura quel était le système institutionnel le plus apte à sa gestion et à permettre cet ajustement sans troquer en échange la sécurité des travailleurs, le débat sur la sclérose des institutions du marché de travail européen et sur la supériorité des capacités d'ajustement du marché américain retrouve de l'actualité.

\footnotetext{
* Organisation internationale du travail, unité des recherches et analyses sur 1'emploi: auer@ilo.org L'auteur remercie Kazutoshi Chatani pour son aide précieuse dans l'exercice de regroupement des pays.
}

Le débat sur l'impact des institutions sur le fonctionnement du marché du travail(1), trouve son origine dans les controverses des années 1980 opposant la souplesse américaine à la sclérose européenne. Un large courant d'idées relie les problèmes européens en matière d'emploi à la rigidité du marché du travail, qui créerait un "fossé» entre insiders et outsiders, les salariés en place défendant leurs «acquis» et les réformes nécessaires du marché du travail en étant entravées. Mais il y a aussi un fort contre-courant à ses idées qui montre que l'impact des institutions sur le fonctionnement du marché du travail n'est pas forcément négatif.

On assiste à la quête des institutions «optimales». $\mathrm{Au}$ sein de ce débat s'est fait jour un nouveau paradigme, devenant pour les pays membres de l'Union européenne un fil conducteur en matière de réformes: la flexicurité. Toutefois, cet oxymore s'accompagne également d'une controverse d'ordre politico-économique.
(1) Le terme « institution» doit s'entendre largement: les appellations «réglementation du marché du travail» ou «politiques du marché du travail» sont souvent utilisées comme synonymes. En effet, elles consistent en un ensemble de réglementations (lois et négociations collectives, coutumes et pratiques), politiques et structures de mise en œuvre régissant à la fois le marché du travail et les conditions de travail et d'emploi des salariés, ayant un impact sur la manière dont les entreprises exercent leur activité. Pour être aptes à forger le comportement des agents, et ce de manière prévisible, ces règles et politiques doivent avoir une certaine durée dans le temps. Les ministères du travail et les services de l'emploi publics ou privés sont des «institutions» du marché de travail au sens propre du terme. 
Cet article trouve sa place dans ce débat et se penche sur la recherche d'institutions optimales de régulation du marché du travail, principalement pour les pays de l'ex-Union européenne à quinze. Seront principalement traitées les questions liées à la législation de la protection de l'emploi (LPE), au système des prestations d'assurance-chômage et aux programmes de politique active de l'emploi. Comme la formulation des règles et politiques du marché du travail dépend crucialement du diagnostic sur les changements intervenus sur ce marché, l'article débattra également de la question de savoir s'il y a ou non une érosion de la relation du travail «standard». Sera ensuite discutée l'option que représente la flexicurité, la question étant en outre posée de savoir si la flexicurité survivra au test de la crise actuelle.

\section{Marchés du travail: en quête de configurations institutionnelles optimales}

Il existe, en théorie économique, un débat sur la configuration institutionnelle optimale des marchés du travail ( $c f$. BlanCHARD, 2005, BlanCHARD, TiRole, 2004). La protection de l'emploi devrait, pour ces auteurs, prendre la forme de taxes de licenciement, plutôt que d'une intervention juridictionnelle. Pour eux, les entreprises connaissent mieux leurs besoins d'adaptation que les juges, mais devraient payer le prix des externalités négatives découlant des licenciements. Il existe également un courant socioéconomique, associé à la recherche sur la flexicurité, qui réfléchit au contexte institutionnel capable de répondre de manière adéquate aux défis que posent les marchés du travail du XXI ${ }^{\mathrm{e}}$ siècle (WiLthageN, 1998, SCHMID, 2002, GAZIER 2003). Les économistes en quête du "contrat de travail unique» forment une sorte de pendant à ceux qui cherchent à définir un contexte institutionnel idéal (CAHUC, Kramarz, 2005).

Les publications théoriques semblent souvent admettre qu'il existe une «manière unique et idéale» de réformer la réglementation du licenciement et de la protection sociale, incarnée par le «contrat unique de travail». Pourtant, dans la pratique, l'opinion que les institutions du marché du travail sont spécifiques à chaque pays semble dominer de plus en plus. Les pays diffèrent et ont évolué différemment, de même que leurs institutions. Il existe une «dépendance liée au parcours» faisant que les réformes radicales sont souvent rares, contrairement aux changements marginaux (2). Ainsi, en dépit des recherches théoriques envisageant des «solutions

(2) Marginal étant souvent défini comme ne s'attaquant pas à la protection des insiders (BOERI, 2005). idéales et uniques », il ne peut exister de système institutionnel unique convenant à tous les cas. De même, il ne semble pas y avoir de modèle institutionnel durable qui soit supérieur à tous les autres. Comme l'observe RoDRIK (1999), «une approche présupposant la supériorité d'un modèle particulier au sein d'une économie capitaliste est une vision très restrictive en termes d'amplitude de variation institutionnelle acceptable (et réalisable) par ces économies de marché». Freeman (1998) traite également de la diversité des caractéristiques institutionnelles et des contextes. Plutôt que de voir un modèle l'emporter, Freeman préconise un dosage savant d'éléments institutionnels à partir d'un éventail de «modèles » devant donner naissance à une nouvelle catégorie institutionnelle, spécifique à chaque pays. BLANCHARD (2005) fait également allusion à cette diversité lorsqu'il écrit: «Ce qui peut sembler idéal pour la Suède ne l'est peut-être pas pour le Chili » (Blanchard, 2005, p. 367)(3).

\section{Croire ou non en l'analyse statistique (économétrique)?}

Ce n'est pas sur ces idiosyncrasies que portent les débats actuels: les économistes du marché du travail préfèrent débattre de questions de «niveau » plutôt que de «structure». Beaucoup s'intéressent à des questions de «niveau» comme: «Les institutions ont-elles un impact sur le marché du travail?», sans se préoccuper plus finement de la structure des institutions, par exemple, à travers des questions comme: "Combien de semaines de préavis sont-elles nécessaires pour garantir un ajustement économique efficace, tout en assurant une ré-allocation des travailleurs également efficace et équitable?». Il s'agira ici au contraire d'établir l'impact que l'ensemble des institutions et réglementations du marché du travail (voir du marché des produits) peuvent avoir sur l'emploi et le chômage.

Les controverses sont très vives. À titre d'exemple, Heckman et Pagés (2000) se livrent à une analyse critique de l'" opinion sensible et prédominante» exprimée selon eux par ABRAHAM, Houseman (1994), Blank, Freeman (1994) et FREEMAN (2000): pour ces derniers, des réglementations (rigides) du marché du travail ne peuvent être tenues coupables des maux du marché de l'emploi. Par contre, Heckman et Pagès montrent que les politiques de la protection de l'emploi ont un impact négatif non négligeable sur le niveau et la répartition de l'emploi en Amérique latine. Dans la même veine, le FMI (2003) conclut que l'Europe gagnerait sur deux tableaux: la croissance du

(3) Il existe également une hypothèse de diversité concernant les «structures et formes» des systèmes nationaux ou groupes de systèmes socio-économiques, tel que le montrent les travaux de Soskice et Hall (2001) ou de Bruno Amable (2005) sur les diversités du capitalisme. 
PIB et la diminution du taux de chômage, si elle adaptait à son marché le faible niveau de réglementation du marché du travail américain. De manière plus radicale encore, la Banque mondiale suggère que l'abondon de nombreuses réglementations du marché du travail aurait pour effet d'améliorer la croissance et l'emploi, de réduire le chômage et la part des activités dites informelles. Le Doing Business Index sur la régulation de l'emploi (employing workers index-EWI) propose un classement selon lequel les pays ayant moins de régulation seraient plus performants que les autres. En illustration, le classement EWI 2009 suggère que des pays comme l'Afghanistan, le Soudan ou l'Iraq auraient ainsi une réglementation plus souple (et donc meilleure) que des pays comme la France, 1'Allemagne, la Suède et d'autres (Doing business, Banque mondiale, 2009). Ceci frôle la bêtise, car le DB/EWI compare 1'incomparable, des pays sans institutions avec des pays à forte institutionnalisation. LAYARD, NICKELL et JACKMANN (1993), estiment qu'il existe bien un certain impact mais sont plus prudents quant à leurs conclusions (4). L'OCDE (1999, 2004, 2006) y voit un impact neutre sur l'emploi et les taux de chômage, convenant toutefois d'un certain impact sur la structure du chômage. D'autres, beaucoup plus critiques, comme BaKer, Glyn, Howell et Schmitt (2005), montrent les limites des études entreprises, comme celle du FMI, et concluent que les impacts des institutions sur le chômage demeurent hautement incertains. Deux études allemandes concluent que les modifications apportées à la législation allemande de protection de l'emploi (seuil d'application des règles porté de 5 à 10 employés) «n'ont eu aucun impact sur l'emploi ou le chômage», mais pourraient en avoir (la question, de l'aveu des auteurs, n'a pas encore été étudiée) sur la structure de l'emploi/du chômage (IzA, 2005).

Pourquoi existe-t-il une telle divergence d'opinions? L'une des explications tient au fait que les résultats sont influencés par le choix des variables indépendantes, par la sélection des pays intégrés dans l'échantillon ainsi que par la période d'observation choisie. Il existe aussi des problèmes de données, mais la dimension idéologique occupe une place importante. Richard FREEMAN (2005) pense que les "préjugés» (priors) expliquent en grande partie ces divergences et que les auteurs suivent leurs idées préétablies et leurs inclinaisons politiques. Il est de fait difficile d'établir la «vérité» en ayant recours à des données et

(4) Leur conclusion vise à dire «que tout compte fait, les lois visant à la protection de l'emploi sont probablement mauvaises pour l'emploi [...] mais qu'il existe toutefois des arguments d'intérêt en leur faveur, la preuve étant que les effets indésirables ou préjudiciables sur l'emploi ne sont pas suffisamment forts pour justifier un abandon total de cette pratique (LAYARD, NiCKELl, JACKMANN, 1993, p. 108). des procédés incertains. Il est toujours possible de «faire parler» les données (introduire des variables indépendantes complémentaires, attribuer ou non un poids à certaines variables, introduire ou retirer des pays, sélectionner une certaine période de temps) ou d'ignorer qu'un lien est statistiquement non significatif et ce, afin que les résultats soient concomitants avec les «préjugés». L'existence d'un préjugé idéologique se voit, par exemple, à travers l'étude empirique menée sur la protection de l'emploi au sein des petites entreprises allemandes, dont il est fait mention ci-dessus: même si aucun impact significatif n'a été décelé quant à la modification des lois sur la protection de l'emploi et sur le chômage, il est préconisé que la protection contre le licenciement en Allemagne soit abandonnée, pour laisser place au principe des indemnités de départ. Parmi les huit cas nationaux étudiés en Amérique latine et aux Antilles par HeCKMAN et PAGÈs (op. cit.), deux d'entre eux seulement venaient étayer leur hypothèse selon laquelle les lois sur la protection de l'emploi sont préjudiciables à l'emploi; dans les autres cas, le lien n'est pas significatif. Toutefois, ils tirent des conclusions générales quant au lien systématiquement négatif existant entre protection de l'emploi et marché du travail (BERG, KUCERA, 2008)(5). Ces deux exemples montrent qu'il peut effectivement exister des «préjugés» forts, qui conduisent ensuite à des changements réglementaires inappropriés.

S'il est difficile de conclure face à un tel débat (avec des idéologies jouant probablement de part et d'autre), les constatations ci-dessus montrent la présence d'opinions contradictoires, en fonction des données, des pays, des méthodes et des modèles utilisés et des préjugés importants. Il conviendrait d'être encore plus nuancé lorsque l'on considère les complémentarités institutionnelles. Par exemple, un haut degré de coordination dans une économie à travers la négociation collective aiderait à annuler tout effet éventuellement négatif des lois sur la protection de l'emploi (ELMESKOV, MARTIN, SCARPETTA, 1998). Les pays affichant un haut degré de coordination pourraient ainsi présenter des marchés du travail efficaces, avec un niveau de la législation sur la protection de l'emploi plus élevé que celui des pays où la coordination est faible. Un effet similaire pourrait découler des éventuelles adaptations internes, plutôt qu'externes, du marché du travail.

Ces incertitudes quant à l'impact de la législation sur la protection de l'emploi sur le fonctionnement du marché du travail sont probablement l'une des raisons pour lesquelles la plupart des

(5) Concernant les autres cas, aucun lien statistiquement significatif n'a été établi entre lois sur la sécurité de l'emploi et travail et chômage. 
réformes, entreprises par un nombre de pays de la zone OCDE, ont été marginales et non radicales, c'est-à-dire affectant les conditions de l'emploi des personnes ayant des contrats de travail flexibles et non pas les insiders avec des CDIs (BoERI, 2005).

Concernant le second élément incriminé comme faussant le libre jeu du marché du travail, la protection sociale liée au non-emploi (prestations d'assurance-chômage, aide et politiques actives du marché du travail), un débat similaire existe. S'y retrouvent ceux qui pensent que la «générosité» des prestations d'assurance-chômage (soit le niveau du salaire de substitution, soit une combinaison du taux du salaire de substitution et de la durée d'allocation des prestations) n'impacte pas de manière négative les niveaux du chômage ou de l'emploi, comme ceux qui pensent au contraire que l'impact est réel. Le premier camp se compose à nouveau d'économistes, tels BAKER, GLYN, HowELL et Schmitт (op. cit.), qui passent en revue une série d'études portant sur l'impact qu'ont les niveaux de salaires de substitution sur le chômage et concluent que l'impact quantitatif est extrêmement peu clair et précis. Au moins, à quelques rares exceptions près, les auteurs cités semblent être très prudents quant à tirer, de leurs constatations incertaines, des conclusions tranchées sur la réforme nécessaire des dispositifs. Coudouel et PACI (2005) passent également au crible certaines de ces publications et concluent que la générosité liée aux prestations d'assurance-chômage semble avoir un effet modéré mais statistiquement significatif sur les niveaux de chômage. L'OCDE (2006) estime que l'impact des niveaux de salaires de substitution sur l'emploi et le chômage s'avère significativement négatif, estimant toutefois également que ces effets négatifs disparaissent lorsque les prestations d'assurance-chômage, pour généreuses qu'elles soient, se doublent de programmes de politique active de l'emploi.

La conclusion à ce débat serait que la durée des versements effectués au titre des prestations d'assurance-chômage aurait un certain impact sur la durée du chômage (6), les niveaux des salaires de substitution ne produisant à eux seuls que des effets marginaux. La complémentarité institutionnelle joue également son rôle et peut, là encore, neutraliser tout effet négatif: par exemple, associer les prestations d'assurance-chômage à des politiques aidant les chômeurs à une recherche de travail intensive

(6) Le rapport de cause à effet n'est toutefois pas clair, une période de chômage qui perdure pouvant tout simplement être due à la prolongation de la cause primaire de la perte de l'emploi, par exemple la clôture d'une entreprise dans une région donnée. L'idée que la prolongation des prestations soit en fait causée par la récession et non pas par le comportement des chômeurs se fait jour aux États-Unis ou la durée des prestations est prolongée lorsque la récession se prolonge, comme actuellement. ou à des politiques d'activation peut effectivement permettre de gérer le «risque moral» auquel le chômeur peut être confronté lorsque le niveau des salaires sur le marché du travail est proche du niveau du montant des prestations.

Mais dans ce cas aussi, les réformes ont été nombreuses, souvent marginales; certaines, telle la réforme Hartz en Allemagne(7) - réforme majeure du marché du travail - ont toutefois permis de réduire considérablement le niveau des prestations d'assurance-chômage octroyées ainsi que leur durée (8).

Il y a également débat sur l'efficacité des politiques actives du marché du travail. À un niveau macro-économique, il peut être indiqué que ces politiques sont efficaces et contribuent à atténuer l'impact de chocs économiques négatifs et défavorables (OCDE, 2006). À un niveau micro-économique, par contre, l'impact de ces politiques est fonction de leurs conceptions, plan et application (Martin, Grubb, 2001; Betcherman et al., 2004; Auer, Efendioglu, LeschKe, 2005).

Il se développe en outre une controverse sur les interactions positives (et parfois négatives) des politiques impliquant une coordination politique complexe (voir ci-dessus) (9). Cette dernière devrait toutefois générer de meilleurs résultats. Par exemple la retraite anticipée, à une époque où elle était encore la principale solution pour réguler les effectifs au sein des grandes entreprises, pouvait contrebalancer l'obstacle de protection contre le licenciement particulièrement fort pour des travailleurs à forte ancienneté. Un autre exemple de politiques combinées réside dans l'impact modérateur que les politiques actives et effectives du marché du travail exercent sur les éventuels effets de trappe à chômage découlant des prestations d'assurance-chômage élevées. De même, les incitations négatives que l'experience rating (10) exerce sur les employeurs peuvent être comptées parmi les interactions à prendre en compte.

(7) La reprise de l'emploi en Allemagne semble seulement marginalement due à la reforme, la plupart des emplois étant créés suite à la forte croissance du secteur de l'exportation et des effets secondaires en découlant.

(8) Ceci ne s'est pas toujours fait en diminuant directement le niveau du salaire de substitution mais, par exemple, en rendant plus sévères les conditions d'accès ouvrant droit à la perception des prestations et/ou en abaissant le salaire de référence servant au calcul.

(9) La complexité accrue des politiques, impliquant la coordination des lignes d'action face aux nombreux intérêts divergents en présence, représente, du point de vue décisionnaire, un défi majeur.

(10) Paiement des contribution à l'assurance chômage en fonction de l'utilisation de celle-ci par les employeurs. 


\section{Les institutions peuvent-elles être un atout?}

Une analyse limitée à l'impact d'une institution ne suffirait sûrement pas à en expliquer le fonctionnement pour le moins complexe. Ce qui est souvent omis dans ce débat, qui souligne en général le coût des institutions, est la contribution positive qu'elles peuvent produire. Faire un petit rappel de certains de ces aspects positifs n'est pas inutile: par exemple, Williamson reconnaissait que les institutions permettent de réduire les coûts de transaction de l'économie et peuvent contribuer à un fonctionnement effectif du marché du travail (ainsi que du marché des produits et services et du marché financier) (Williamson, 1985). En l'absence de telles institutions, les coûts de transaction (par exemple, embauche, sélection et licenciement) sont au final plus élevés que les coûts liés à la protection de l'emploi. En analyse micro-économique, la sécurité de l'emploi, découlant entre autre du travail des institutions, peut s'avérer positive pour la productivité du fait qu'elle incite les entreprises à sélectionner minutieusement les salariés ainsi qu'à les former. Par exemple, MARINESCU (2006) a montré, dans le cadre de la législation britannique du travail, que le fait de revenir à une «période d'essai»(11) d'un an (d'abord portée à deux ans par le gouvernement Thatcher), avait des résultats positifs pour les employeurs en termes d'adéquation de l'offre et de la demande du travail, sans effets négatifs sur l'emploi. Mais les institutions doivent également satisfaire à des buts d'équité : les prestations d'assurance-chômage devraient garantir un revenu aux personnes sans emploi et les politiques actives du marché du travail permettre d'accélérer leur réintégration. L'OCDE (2006) reconnaît que les politiques actives du marché du travail ont pour effet d'atténuer considérablement l'impact des chocs macro-économiques négatifs et défavorables. Les prestations et indemnités perçues par les personnes sans emploi servent à leur tour à soutenir la consommation et, partant, la croissance, les dépenses sociales étant simultanément légitimées par les motifs économiques et sociaux.

La législation de protection de l'emploi protège également les salariés. Peu de chose démontre que l'abandon des systèmes de protection entraînera pour les salariés en marge des gains nets en termes d'emploi et de chômage. La protection de l'emploi réduit les entrées en chômage, alors qu'en même temps elle tend à réduire également les entrées en emploi. En partant de ce postulat, une amélioration de la performance du marché du travail, pour les salariés en marge, coïncide presque à coup sûr avec une détérioration des conditions pour les insiders.

(11) Ce point fait référence à la période précédant celle où un employeur peut se voir poursuivi pour licenciement abusif.
Pendant la plus grande partie de l'après-guerre, des tentatives furent faites pour instituer, en tant que norme, des relations de travail basées sur des contrats stables, à temps plein. Aujourd'hui, beaucoup craignent que cette norme ne soit progressivement remplacée par un contexte où ne prévaudraient que des emplois flexibles et emplois à court terme, surtout pour les femmes.

\section{La fin des emplois stables?}

La mondialisation est censée générer des marchés du travail plus souples et plus dynamiques. Mais cette nouvelle flexibilité nécessite de nouvelles formes de sécurité allant bien au-delà des limites d'une simple entreprise. Selon RoDriK (1999), «une économie moderne de marché est une économie où le changement est constant et où le risque idiosyncrasique (soit spécifique à chaque individu) lié aux revenus et à l'emploi est omniprésent. La croissance économique moderne impose une transition, le passage d'une économie statique à une économie dynamique, où les tâches effectuées par les salariés évoluent constamment [...]. Et les risques contre lesquels il convient de se prémunir deviennent alors, selon le mode traditionnel, bien moins gérables lorsque les marchés croissent et s'étendent». La plupart des observateurs notent des changements spectaculaires dans la relation de travail, certains y voyant même la fin du travail (salarié) (RIFKIN, Heilbroner, 1995, Beck 2000) ou, du moins, un système évoluant «au-delà de l'emploi» (SuPIOT, 1999).

Les récessions, réductions d'effectifs, le chômage et les emplois précaires observables donnent à penser que le fait de détenir un emploi durable et à plus long terme est l'exception plutôt que la règle, et qu'en présence de cette phase de la mondialisation, la flexibilité (numérique) a finalement eu le dessus.

Toutefois, ce postulat doit pouvoir résister au test de la preuve empirique. Tous les pays de l'OCDE comptent encore une part importante des contrats de travail de longue durée ( $c f$. OIT, 1996; OCDE, 1999; Auer, Cazes, 2003; Neumark, 2000). Même s'il y a une grande variance par pays, secteurs, groupe de salariés dans ce pourcentage, les systèmes d'emploi ont un taux de stabilité remarquable comparé au spectre du «tout est devenu flexible». Cette stabilité a été récemment confirmée par des études comparatives nationales et internationales, (ERLINGHAGEN, Knuth, 2004; Doogan, 2004 ; Souza-Poza, 2004). STEVENs (2005) montre que même aux États-Unis, où la tenue moyenne d'un poste est notablement plus faible, les «relations de travail de longue durée, liant le salarié à un seul et unique employeur, sont une caractéristique majeure du marché américain du travail. Ceci était le cas en 2002, l'étant d'ailleurs tout autant qu'en 1969. [...] Un peu plus de la moitié 


\section{Graphique 1: Ancienneté moyenne dans l'Union européenne à quinze en 1992 et 2005}

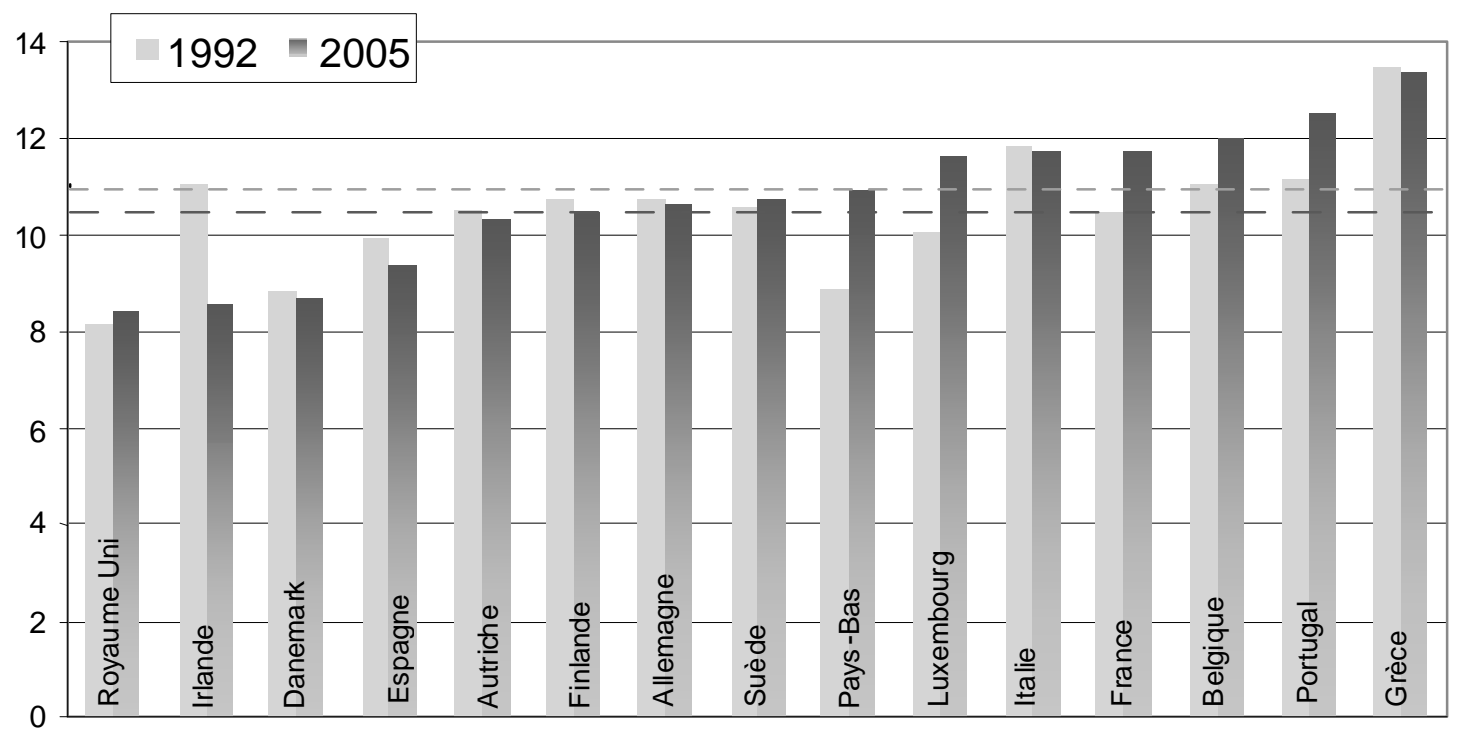

Source: calculs propres effectués à partir des bases Eurostat.

Note de lecture: classement 2005; les lignes en pointillé correspondent à la moyenne de l'Union européenne: 10,48 pour 1992 et 10,74 pour 2005.

Des chiffres quelque peu comparables pour les États-Unis donnent 6,7 années d'occupation des postes, pour 1992, et 6,9 pour 2004. La durée moyenne d'occupation des postes au Japon était de douze ans en 2000 (Source: États-Unis: Bureau des statistiques du travail, Japon: ONo, 2006).

des salariés hommes, achevant leur carrière professionnelle en 1969, avait passé au moins 20 ans auprès du même employeur; la même constatation vaut en $2002 »$ (pp. 1 et 24).

Si cela est le cas sur le marché du travail très flexible des États-Unis, ceci doit l'être d'autant plus sur les marchés européens. De fait, comme le montre le graphique 1, la stabilité de l'emploi mesurée par l'ancienneté moyenne de ceux ayant un emploi n'a quasiment pas changé sur la période allant de 1992 à 2005. Les données pour 2006 confirment cette stabilité «en moyenne».

Hormis l'Irlande, la plupart des pays affichent des valeurs stables ou en augmentation, portant la moyenne européenne non pondérée de 10,48 années en 1992, à 10,74 en 2005. Les pays dont l'ancienneté moyenne est plus faible sont également ceux dont la flexibilité numérique est plus élevée, comme le montre le cas des États-Unis et du Royaume-Uni, mais également du Danemark. Par contraste, la majeure partie des pays figurant en partie droite du graphique 1 sont ceux connus pour appliquer une réglementation stricte en matière de licenciement (OcDE, 2004).

Toutefois, si la moyenne demeure inchangée, les différents éléments la composant ont vu des modifications s'opérer. Ces changements portent sur le sexe, l'âge, la composition du secteur économique, etc. Par exemple, l'ancienneté moyenne des femmes augmente, alors que celle des hommes diminue. Il existe également une légère augmentation du nombre des postes occupés pendant une période plus courte
( $<1$ an) ainsi qu'un léger déclin concernant les postes occupés pendant une durée plus longue ( $>10$ ans), le recul du nombre des postes à forte ancienneté étant particulièrement marqué en Irlande (moins 13\%).

Des changements sont intervenus, et l'étude de la structure du cycle économique et de l'âge le confirme: les jeunes employés sont systématiquement confrontés à des périodes de travail plus courtes et la flexibilité est (et a toujours été) un phénomène ciblant principalement les jeunes gens. En 2001, la durée moyenne d'occupation des postes n'était que deux ans seulement pour la tranche d'âge des 14-24 ans, huit ans pour les 25-44 ans et dix-sept ans pour les plus de 45 ans (12).

En dépit de ces changements, le contrat de travail de longue durée reste la forme principale de l'emploi. Cette forme du travail cependant inclut de manière de plus en plus prononcée l'emploi à temps partiel. L'Enquête européenne sur la main-d'œuvre révèle qu'environ $85 \%$ des emplois à temps plein et temps partiel en Europe sont des contrats à durée indéterminée.

Le changement le plus notable intervenu dans le cadre des contrats-types de travail (Bosch, 2002) consiste dans l'augmentation de la participation active des femmes et dans l'accroissement parallèle des emplois à temps partiel. Nombre d'emplois à temps partiel toutefois correspondent à

(12) Pour les jeunes les écarts entre les pays reflètent aussi des différences dans la durée de la scolarité. 
Graphique 2 : Rapport entre ancienneté au travail et productivité dans treize États membres 1992-2002

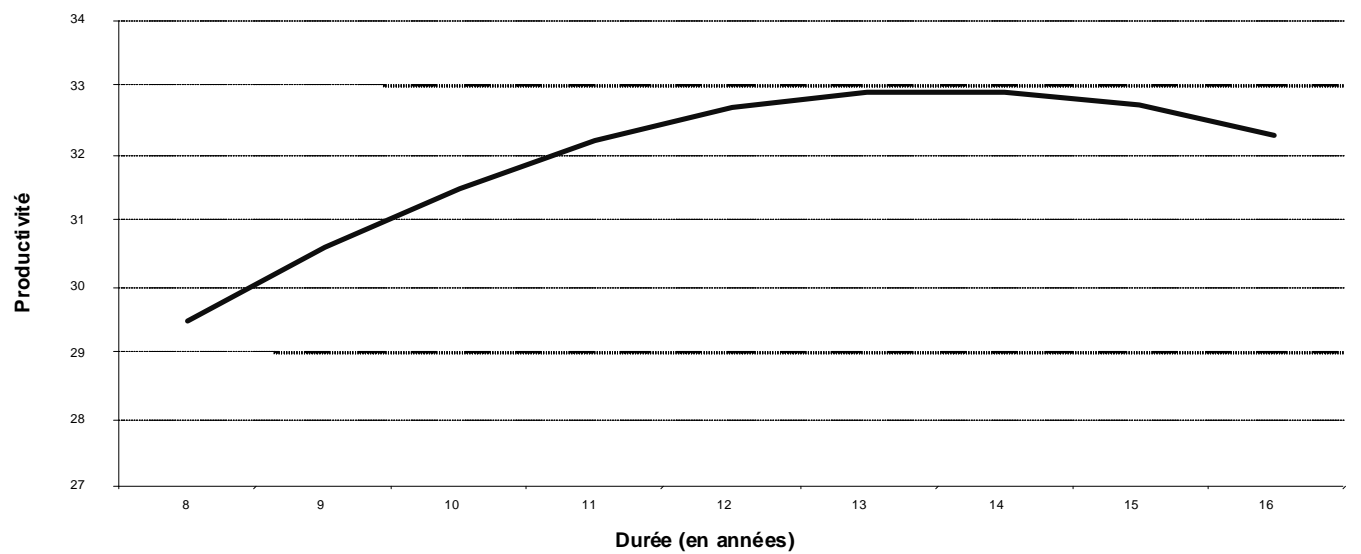

Source: données issues des bases Eurostat, pour la période 1992-2002.

Note de lecture : productivité = production horaire, en euros; durée d'occupation du poste/ancienneté = durée moyenne pour tous ceux en emploi dans six secteurs et treize pays européens (Union européenne à quinze sans l'Irelande et l'Autriche) en années. Source: Auer, Berg, Coulibaly (2004).

une modification des préférences, côté offre(13). L'augmentation notable des emplois à temps partiel en Europe (passant de $14 \%$ de l'ensemble des emplois au sein de l'Union européenne en 1992, à plus de $19 \%$ en 2005) s'accompagne d'une croissance plus que disproportionnée du nombre des emplois de longue durée à temps partiel (+ 10 ans) (DoOgAn (2005).

\section{Les emplois stables sont-ils de bons emplois?}

Cependant, les emplois stables ne sont pas toujours synonymes de bons emplois. L'OCDE montre qu'environ $10 \%$ des emplois temporaires (soit approximativement 1,5\% de tous les emplois) sont des emplois temporaires à long terme avec une durée cumulée de cinq ans et plus.

Nos observations empiriques ne permettent pas de conclure qu'il existe une érosion générale de la relation de l'emploi induite par la demande, ni par l'offre. En termes de qualité d'emploi et de sécurité d'emploi, il existe probablement un décalage de plus en plus grand entre les postes proposés par les employeurs (offres d'emploi, demande de travail) et les postes recherchés par les travailleurs (offre de travail, demande de l'emploi). Les mutations en cours auront probablement pour effet de creuser encore le fossé entre les attentes de part et d'autre. Il faudrait

(13) La modification des préférences, côté offre, peut se fonder sur le taux de choix volontaire lié aux emplois à temps partiel, en particulier pour les femmes, ce taux variant d'un pays à l'autre: ce taux est bas, représentant environ 4,5\% au Luxembourg et aux Pays-Bas, de plus de $30 \%$ en Espagne, France et Italie, et supérieur à $40 \%$ au Portugal et en Grèce. donc s'attendre très probablement non pas à une course visant les extrêmes, mais à un nouvel «équilibre» entre emplois flexibles et emplois stables, les premiers gagnant quelques points de pourcentage au détriment des seconds. La crise financière actuelle peut par contre avoir un impact sur ce nouvel équilibre en faveur de l'emploi flexible.

\section{La stabilité de l'emploi est bénéfique à la productivité... jusqu'à un certain point}

Le débat sur les avantages de la flexibilité suppose généralement que la performance et la productivité du marché de l'emploi bénéficieront toutes deux des capacités accrues d'adaptation des marchés du travail. L'argument est que la ré-affectation plus rapide des travailleurs entre secteurs moins productifs et secteurs plus productifs aura pour effet d'accroître la productivité globale. Si ceci peut être considéré comme vrai, un point fait cependant défaut à l'analyse : tous les postes ainsi réaffectés ne présentent pas une productivité plus élevée. De plus, les emplois ne sont pas constamment redistribués entre les secteurs. La stabilité sur les marchés du travail reste importante: les entreprises rentables savent s'adapter, en interne, et utiliser le potentiel de leurs effectifs en place. Les études micro-économiques accordent ainsi beaucoup d'importance à la sécurité de l'emploi (emplois stables et permanents), à la fois en termes d'augmentation de salaire et d'investissements en formation. Dire où se situe le seuil de rentabilité, soit un point d'équilibre entre «trop» et «pas assez» d'ancienneté, est difficile à déterminer, mais nos propres études sur la question ont révélé qu'une ancienneté considérable peut aller 
de pair avec une forte productivité, tandis qu'une trop grande flexibilité, ou une stabilité trop importante, peut peser sur la productivité. Notre modèle économétrique montre qu'au niveau agrégé pour tous ceux qui ont un emploi, dans treize pays européens, le point d'inflexion où la productivité commence à décroître est de près de quatorze ans d'ancienneté (Auer, Berg, Coulibaly, 2004).

Ces constatations représentent une durée moyenne d'ancienneté. Par secteur, métier ou par pays, et pour d'autres périodes de temps, elles peuvent varier. Par ailleurs, ce point d'inflexion, résultante des données agrégées, ne peut être interprété comme constituant la productivité maximale d'un employé individuel. En d'autres termes, s'il peut en théorie exister une «durée optimale de l'ancienneté au travail», ce point ne peut être précisément indiqué. Les essais empiriques montrent que les postes occupés pendant une durée très courte (moins d'un an) et ceux occupés pendant une très longue durée (en particulier pour des durées supérieures à vingt ans) peuvent avoir des effets négatifs sur la productivité. Les études menées à une échelle micro-économique aboutissent aux mêmes conclusions: pour KRAMARz et Roux (1999), les entreprises françaises, ayant un nombre élevé de salariés occupant leurs postes depuis quatre à dix ans, sont plus productives que celles présentant une forte proportion de postes dont la durée d'occupation est soit inférieure à quatre ans soit nettement supérieure à dix ans.

Contrairement à l'idée que les secteurs les plus innovants ont une flexibilité numérique très grande de leurs effectifs, l'emploi stable semble également présent dans ces secteurs: PACELLI et al. (1998), dans une étude portant sur 2800 entreprises italiennes, menée sur la période de 1985 à 1991 montrent que l'emploi est plus stable dans les industries innovantes que dans les industries du secteur traditionnel. De même, Michie et SHeEHAN (2003), dans une étude sur l'industrie du Royaume-Uni, concluent que le faible taux de rotation de la maind'œuvre et la polyvalence sont en corrélation positive avec l'innovation.

La question se pose de savoir si la flexibilité interne fonctionnelle et la flexibilité numérique externe constituent des choix alternatifs d'adaptation de la main-d'œuvre au changement structurel. Tandis que CAPELLI et NeUMARK (2004) s'attachent à démontrer que les entreprises américaines, efficaces et rentables, utilisent toute forme de flexibilité, et qu'il n'existe aucun signe clair d'un tel trade-off; dans d'autres pays, les formes d'adaptation interne et externe ont bel et bien tendance à se substituer l'une à l'autre. Un exemple en est la disposition prise chez Volkswagen, en Allemagne, en faveur des réductions du temps de travail de préférence à des licenciements. Abraham et Housemann (1995), dans une comparaison des formes d'adaptation des effectifs en Allemagne et aux États-Unis, montrent que si l'ampleur de l'adaptation est comparable, la préférence va en Allemagne à l'adaptation interne (aidée en cela par un système de chômage partiel) plutôt qu'à l'adaptation externe; aux États-Unis, l'adaptation externe, avec mise au chômage complet, est la solution retenue. Une étude allemande (HoHendanNer, Bellman, 2006) montre qu'environ $80 \%$ des entreprises de toutes tailles ont recours aux adaptations internes plutôt qu'externes.

\section{La stabilité de l'emploi n'a pas pour résultat la sécurité de l'emploi: un paradoxe}

La question demeure toutefois de savoir si une telle stabilité au sein des systèmes de travail confère également la sécurité de l'emploi. Des emplois stables apportent plus de sécurité que des emplois non stables et de courte durée, et la perception de sécurité des travailleurs est moindre pour les emplois temporaires que pour les emplois stables: des emplois dans le secteur public sont perçus comme conférant plus de sécurité (CLARK, PostelVINAY, 2006). Toutefois, au sein du secteur privé, la protection plus élevée qu'offrent en principe les emplois stables ne correspond pas toujours à une perception accrue de la sécurité de l'emploi. La corrélation simple entre l'ancienneté et la perception de la sécurité de l'emploi (voir graphique 2) ne vient pas corroborer le rôle de renforcement de sécurité qu'offre une protection plus stricte. Le lien qui se dégage est faible et statistiquement non significatif, pointant même dans la mauvaise direction (suggérant en tout cas, qu'il ne suffit pas d'occuper un poste stable pour se sentir en sécurité). Il serait possible d'arguer que la proportion des emplois à temps partiel tend à renforcer la perception d'insécurité; toutefois, la corrélation avec la perception de sécurité de l'emploi s'avère positive et non négative. Les salariés aux Pays-Bas (où le nombre des emplois à temps partiel est le plus élevé de l'Union européenne) ont un sentiment de plus grande sécurité que les salariés en Grèce où la part des emplois à temps partiel est pourtant plus faible. La perception subjective liée à la sécurité de l'emploi ne semble pas uniquement déterminée par l'ancienneté sur un poste. Pour exemple, la corrélation entre la perception de la sécurité et le taux de chômage est très significative: la conjoncture économique générale influence fortement les sentiments subjectifs. Au Japon, la perception d'insécurité liée à l'emploi est forte en 2000, probablement du fait de la longue récession que traverse le pays; pourtant, l'ancienneté moyenne des travailleurs est parmi les plus longues de tous les pays étudiés. 
Graphique 3 : Insécurité d'emploi et durée d'occupation des postes en 1996 (gauche) et 2000 (droite)
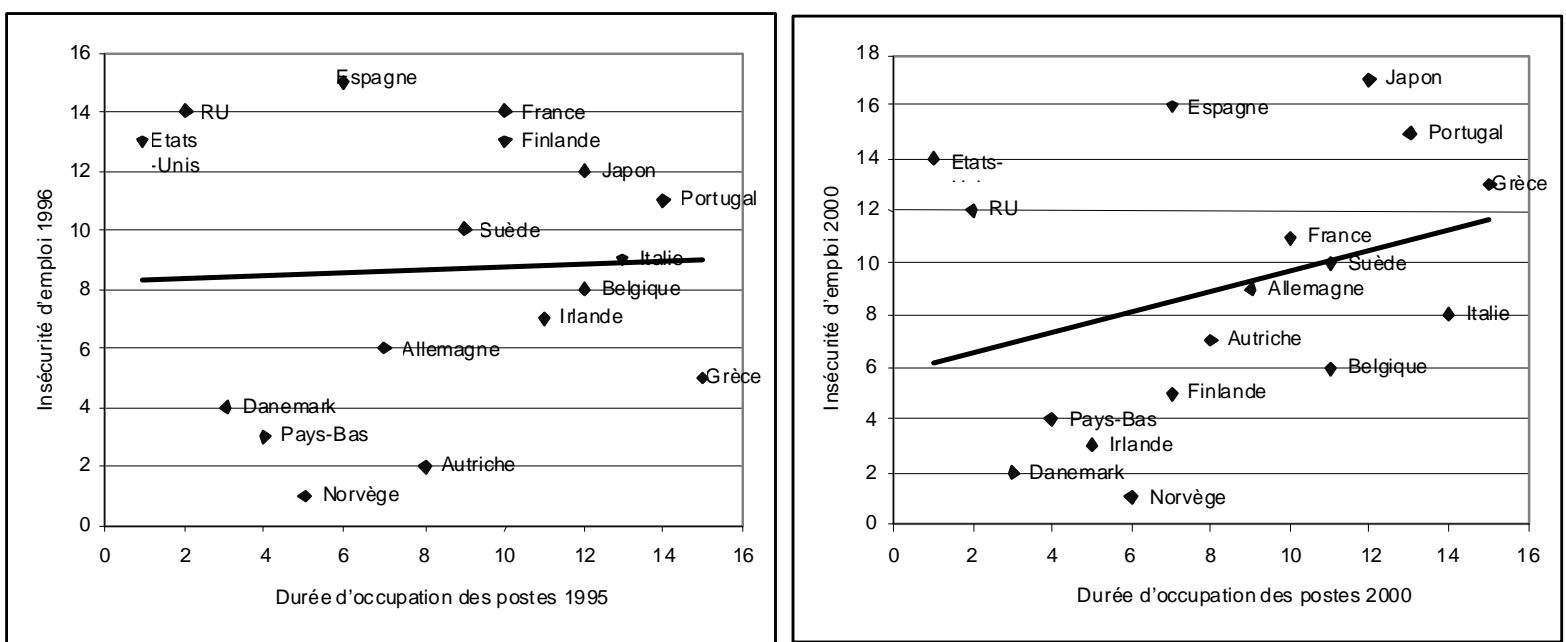

Source: données fournies par: l'International Survey Research (ISR), l'OCDE, Eurostat et les sources nationales.

Note de lecture: les coefficients sont non significatifs (sont indiqués, dans les deux graphiques, les classements par pays: sécurité $1=$ nombre le plus important de salariés reconnaissant un sentiment de sécurité; durée d'occupation des postes 1 = durée la plus courte d'occupation des postes).

Ces résultats, paradoxaux, montrent que l'ancienneté n'influe apparemment pas systématiquement sur les anticipations liées à la sécurité de l'emploi. Bien que la sécurité de l'emploi joue un rôle très significatif dans la perception de la satisfaction professionnelle (COMMISSION EUROPÉENNE, 2001, European Foundation, 2007), il ne se dégage pourtant aucun schéma clair. Dans certains pays, l'ancienneté est comparativement faible, avec toutefois une perception élevée de la sécurité de l'emploi et un grand nombre d'emplois qualitatifs; dans d'autres, l'ancienneté est élevée, mais la part des emplois qualitatifs faible, la sécurité perçue également (AUER, 2007).

À première vue, ceci semble indiquer que l'ancienneté écoulée n'est pas un bon indicateur de la perception subjective de la sécurité de l'emploi. Pour L'OCDE (2004, p. 92), la rigueur de la protection de l'emploi (LPE), en forte corrélation avec l'ancienneté, ne véhicule pas le sentiment de sécurité qu'elle est supposée véhiculer. Cependant, les salariés ressentent une sécurité nettement accrue à occuper des emplois permanents, dans le secteur public, et témoignent d'une réelle insécurité lorsqu'ils occupent des emplois temporaires (14). La relation négative entre ancienneté et sécurité perçue est également confirmée lorsque l'on s'appuie sur la seconde (1995-1996) et la quatrième (données pour 2005) enquête européenne sur les conditions de travail. Elles montrent que l'insécurité perçue,

(14) Dans une étude récente, se fondant sur des données tirées du panel européen des ménages, Clark et Postel-VInAY (2006) montrent que le rôle non sécurisant de la législation en matière de protection d'emploi (ou de l'ancienneté), vaut en particulier pour les emplois permanents du secteur privé, ces derniers formant le plus gros des emplois. face à l'emploi, semble plus forte parmi les salariés des pays avec une ancienneté élevée. Ce constat ne permet pourtant pas de dire que la protection de l'emploi est inefficace pour apporter un sentiment de sécurité et qu'il faudrait la remplacer par une sécurité sociale déliée de l'emploi. Imaginons pour un instant un système d'emploi qui n'offre aucune protection contre le licenciement mais uniquement une protection sociale en dehors des entreprises. $\mathrm{Ce}$ contrefactuel montrerait très probablement que la perception de sécurité de l'emploi sera amoindrie. On ne peut imaginer une protection sociale conséquente que comme un complément à une protection de l'emploi. Mais il apparaît qu'en maximisant la protection de l'emploi, on n'arrive pas aux résultats escomptés en terme de sécurité perçue.

Le contexte mondial actuel renforce le sentiment d'insécurité et d'insatisfaction dans le travail: les salariés peuvent avoir besoin d'une certaine mobilité ascendante (sentiment de progression dans l'échelle sociale) pour ressentir plus de sécurité et de satisfaction.

Il ressort de cette analyse que les pays dont la flexibilité numérique est la plus élevée sont aussi ceux qui affichent les meilleurs taux dans la conversion de postes temporaires en postes permanents et de postes peu qualitatifs en postes plus qualitatifs (voir CoMmission EUROPÉENNE, 2001, 2004). De même, les pays qui ont un type de sécurité que l'on peut qualifier «de marché de travail» (une combinaison entre sécurité de l'emploi dans l'entreprise et une sécurité sociale active en dehors de l'emploi) semblent parvenir à un meilleur degré de protection que des pays qui tablent sur une sécurité de l'emploi seule. 
Graphique 4 : Insécurité d'emploi et politiques du marché du travail, 2000

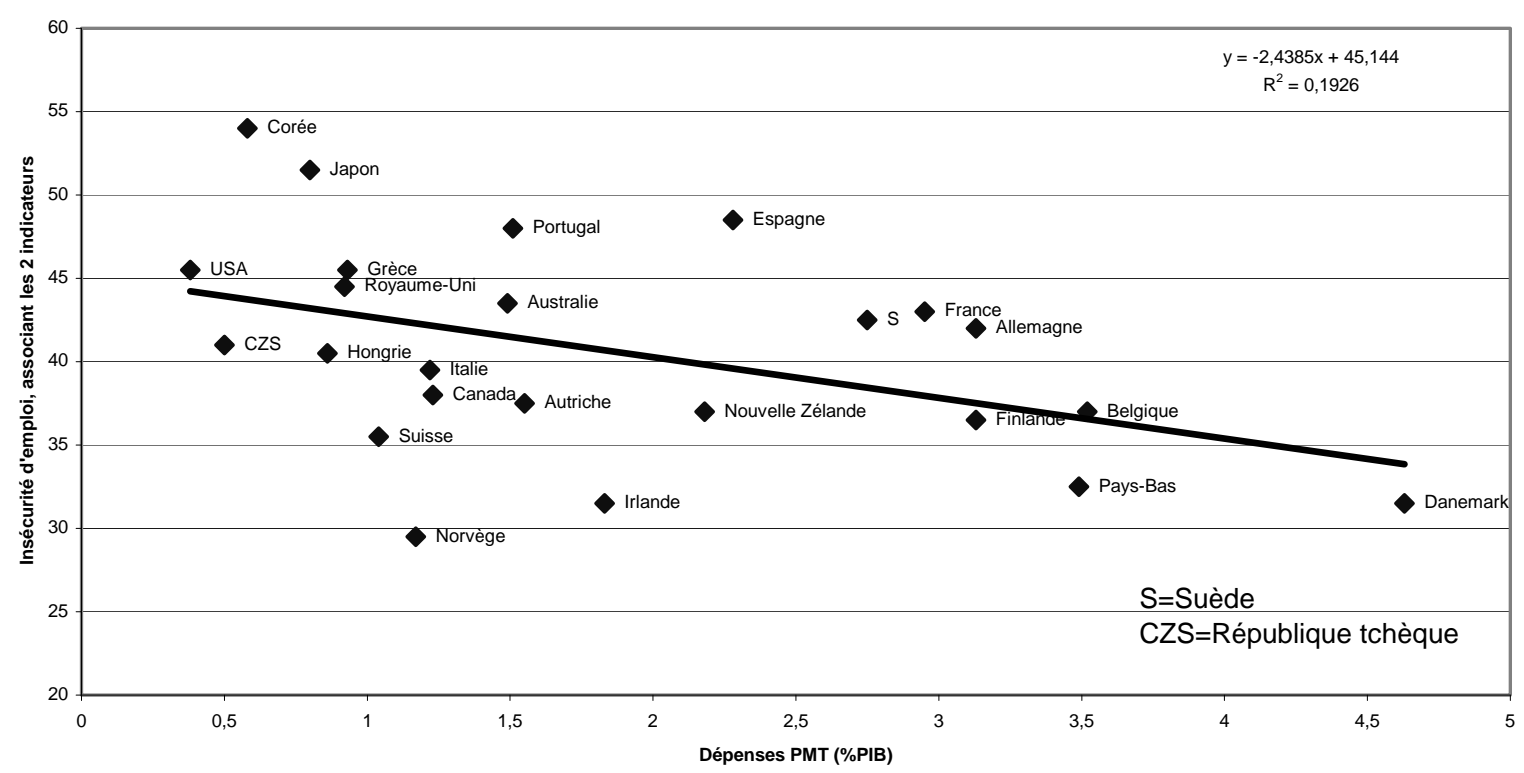

Sources: données sur l'insécurité de l'emploi, émanant de l'ISR, citées dans les publications de l'OCDE, 2001; données relatives aux dépenses PMT, émanant de l'OCDE 2004, données 2001 pour l'Irlande).

Note de lecture: l'insécurité de l'emploi est le pourcentage moyen se dégageant des deux indicateurs: 1) salariés inquiets de l'avenir de leur entreprise et 2) ceux non sûrs de conserver un emploi au sein de l'entreprise, même si leurs performances personnelles sont bonnes.

\section{Graphique 5: Regroupement des pays par degré de flexibilité et de sécurité}

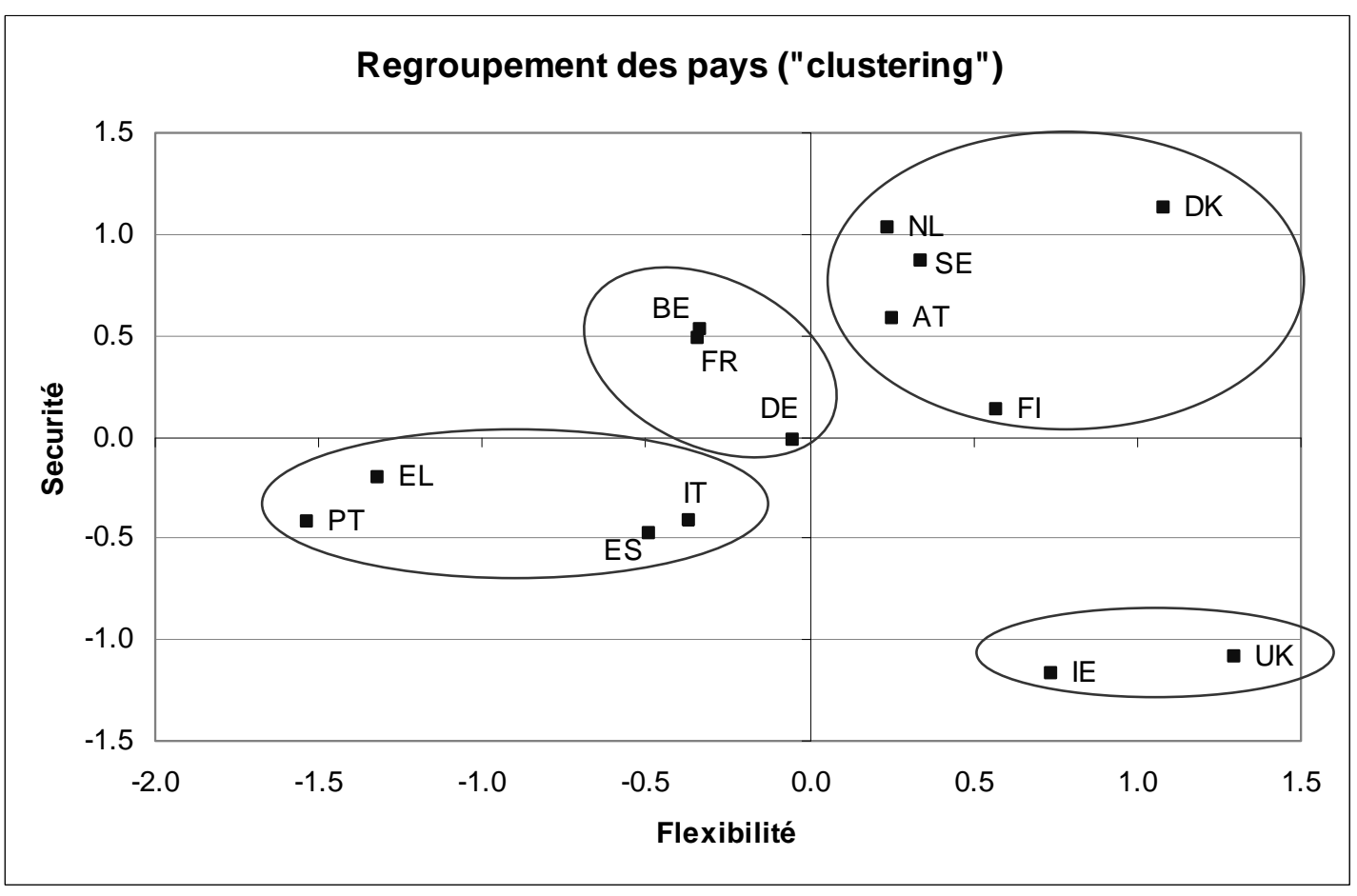

Sources: Fondation européenne à Dublin, OCDE, Eurostat, Commission européenne).

Note de lecture: le regroupement des pays est fait sur la base de huit variables de flexibilité et de sécurité. Flexibilité interne: flexibilité du temps de travail (2000) et organisation du travail moderne (2004); flexibilité externe: ancienneté moyenne dans l'entreprise (2006); protection légale contre le licenciement pour travailleurs réguliers et temporaires (2003); sécurité: dépenses pour la politique de l'emploi (active et passive) par $1 \%$ des chômeurs (moyenne 2000-2005), dépense pour la protection sociale (moyenne 2000-2004) et part des travailleurs couverts par les accords collectifs (2004).

(Standardisé à l'aide de «z-scores» avec un poids égal donné à tout indicateur). 
La protection sociale sur le marché du travail (en fait le système d'indemnisation du chômage et la politique active de l'emploi) joue bien évidemment un rôle clé pour accroître la sécurité sur les marchés $\mathrm{du}$ travail. Le graphique 4 montre la relation entre l'insécurité de l'emploi perçue, et les dépenses engagées en termes de politiques du marché du travail (PMT). Il existe de fait un rapport négatif entre ces deux variables, tendant à confirmer le rôle de renforcement de la sécurité qu'ont les politiques du marché du travail, ceci étant confirmé par l'analyse de l'OCDE $(2004,2006)$ ainsi que par CLARK et Postel-Vinay (2006).

\section{Différents systèmes de l'emploi}

Depuis la parution de l'ouvrage Les trois mondes $d u$ capitalisme $d u$ bien-être (1999), d'Esping Andersen, ou de ceux d'Albert Capitalisme contre Capitalisme (1991), Hall et Soskice Variétés du capitalisme (2001) ou d' Amable Les cinq capitalismes diversité des systèmes économiques et sociaux dans la mondialisation (2005), le regroupement (cluster) des pays, par «régimes», est à la mode. Les efforts de recherche ont porté sur la détermination des regroupements fiables des pays. Ce travail a été étendu à des domaines plus restreints comme la «flexicurité» (Commission Européenne, 2006, 2007). Le degré de sophistication de ces regroupements varie du simple au complexe. Le graphique 5 utilise huit variables pour mesurer le dégrée de flexibilité ou de sécurité.

Ce regroupement montre que des pays comme le Danemark, la Suède, l'Autriche, les Pays-Bas et la Finlande peuvent être considérés comme ayant établi un certain équilibre entre flexibilité et sécurité, tandis que d'autres préfèrent soit plus de sécurité sans trop de flexibilité, soit une flexibilité sans trop de sécurité. Si on considérait ce regroupement comme une variable expliquant les résultats du tableau 1 ci-dessous, on pourrait conclure que les pays qui ont établi cet équilibre entre flexibilité et sécurité sur leurs marchés du travail montrent de meilleurs résultats que les autres. Ce résultat donnerait donc une certaine légitimité empirique aux politiques inspirés par la flexicurité. Bien que ces résultats soient donnés principalement à titre illustratif, ils indiquent une tendance se recoupant en partie avec beaucoup d'autres rapports (voir Commission EUROPÉENNE, 2006, 2007), mais il paraît difficile d'établir sur cette base une causalité robuste. Il est vrai que nous observons de meilleurs résultats dans les pays de flexicurité, mais ceci pourrait seulement montrer que des succès antérieurs de ce pays leur ont permis d'établir des institutions du marché de travail «optimales». Il faut aussi dire que certaines de ces pays avaient des performances d'emploi très mauvaises avant de reformer leurs marchés de travail dans un sens de «flexicurité» (notamment le Danemark et les Pays-Bas) (Auer, 2000) ce qui confirmerait la relation positive constaté.

\section{Tableau 1 : Indicateurs de résultat par pays en fonction de l'équilibre entre flexibilité et sécurité sur leur marché du travail}

\begin{tabular}{|l|c|c|c|}
\hline & $\begin{array}{c}\text { Pays ayant } \\
\text { un degré } \\
\text { élevé de } \\
\text { flexicurité } \\
\text { (5) }\end{array}$ & $\begin{array}{c}\text { Autres } \\
\text { pays (10) }\end{array}$ & $\begin{array}{c}\text { Différence } \\
\text { en points }\end{array}$ \\
\hline $\begin{array}{l}\text { Taux d'emploi } \\
\text { 15-64 (TE) }\end{array}$ & 72,0 & 63,1 & $+8,9$ \\
\hline $\begin{array}{l}\text { TE en équiva- } \\
\text { lent temps plein } \\
\text { (TEETP) 15-64 }\end{array}$ & 64,2 & 58,2 & $+6,0$ \\
\hline TE Femmes & 66,9 & 53,8 & $+13,1$ \\
\hline EETP Femmes & 55,6 & 46,9 & $+8,7$ \\
\hline Taux de chômage & 5,4 & 7,2 & $-1,8$ \\
\hline $\begin{array}{l}\text { Productivité/heure } \\
\text { en euros }\end{array}$ & $29,40 €$ & $27,40 €$ & $+2,00 €$ \\
\hline $\begin{array}{l}\text { Productivité/heure } \\
\text { (2000=100) }\end{array}$ & 110,3 & 107,6 & $+2,7$ \\
\hline Coefficient Gini & 25,1 & 30,6 & $-5,5$ \\
\hline $\begin{array}{l}\text { Effectivité de la } \\
\text { protection sociale }\end{array}$ & 14,5 & 8,4 & $+6,1$ \\
\hline $\begin{array}{l}\text { Dépenses pour } \\
\text { la politique de } \\
\text { l'emploi par 1\% } \\
\text { des chômeurs }\end{array}$ & 0,63 & 0,28 & $+0,35$ \\
\hline
\end{tabular}

Source: Commission européenne, BIT, OCDE, données pour 2000-2006.

Des mises en garde s'imposent: le regroupement par pays (quels que soient les critères y présidant) n'a qu'une valeur d'illustration et ne résisterait probablement pas à une analyse plus poussée. Dès lors que leurs traits ou facteurs communs sont soumis à un examen minutieux, il est avéré que tous les groupes (comme ceux d'Esping Andersen) seraient dans l'incapacité de résister au test de «similitude». Les variations se dessinant en effet au sein même de ces groupes sont importantes.

Le Danemark et les Pays-Bas peuvent servir d'exemple. Ces deux pays sont considérés comme des prototypes de flexicurité en raison de leur combinaison «protection de l'emploi/sécurité sociale». Mais les travailleurs danois affichent une ancienneté au travail relativement peu élevée et une proportion moyenne d'emplois à temps partiel, tandis qu'aux Pays-Bas l'ancienneté au travail est plus longue et les emplois à temps partiel beaucoup plus nombreux. En termes d'ancienneté, ces pays ont également évolué différemment: l'ancienneté a augmenté aux PaysBas, et légèrement décru au Danemark. Toutefois, les performances du marché du travail sont bonnes pour ces deux États. Même un regroupement encore plus poussé, faisant par exemple appel à une analyse en composantes principales (COMMISSION EUROPÉENNE, 2006, 2007; OCDE, 2006), ne peut éviter cet écueil. Que les méthodes d'analyse soient simples ou complexes, le regroupement est extrêmement sensible au choix des variables et il n'existe aucun exemple qui puisse traiter à la fois toutes les variables pertinentes. C'est peut-être là également une base empirique pour l'af- 
firmation de la Commission Européenne qu'il existe de nombreuses versions différentes de la flexicurité (no-size-fits-all). Certes, cette affirmation est aussi due à la résistance des pays à être assujettis à un quelconque modèle unique de flexicurité. Si le regroupement pose problème - la classification des pays étant clairement sensible au choix des variables - le fait d'avoir recours aux indicateurs de flexibilité du marché du travail montre que les pays les plus flexibles ne sont pas ceux dont la performance est la meilleure; les pays affichant une protection moyenne de l'emploi, associée à une protection sociale forte, sont en tête des résultats. Au sein de ces pays, on peut parler de l'intégration des politiques sociales et économiques, ainsi que d'une mobilité protégée ayant pour résultat une sécurité du marché meilleure que dans les pays «flexibles», mais aussi des pays qui tablent plus sur la protection de l'emploi.

\section{Flexicurité, une autre organisation du marché du travail?}

Nous pouvons donc, pour les pays du quadrant nord-est du graphique 1, parler d'une flexibilité ne se faisant pas au détriment de la sécurité des salariés, ceci du fait que les prestations d'assurance-chômage et les politiques actives du marché du travail, de même que les droits sociaux, tels les congés parentaux et congés de formation, ont pour résultat ce que certains appellent la «flexicurité» (Wilthagen, 1998, MADSEN, 2003), d'autres la «mobilité protégée» (AUER, 2005), d'autres encore "l'équilibre entre flexibilité et sécurité» (CAzes, Nesporova, 2003) ou encore les «marchés transitionnels du travail» (GAZIER, 2003; SCHMID, 2002; Auer, Gazier, 2006). L'Union européenne a récemment fait sienne les «principes communs de flexicurité »(15). Ce terme est toutefois l'objet de débats dans de nombreuses parties du monde, comme en Inde (Shyam Sundar, 2005), en Chine ou en Amérique latine.

Sous sa forme la plus simple, l'approche de la flexicurité préconise une négociation des partenaires sociaux pour trouver un équilibre entre une protection de l'emploi et une protection sociale qui permette à la fois l'ajustement aux contraintes de la mondialisation et de la concurrence mondiale et une sécurité des travailleurs. Des droits sociaux devraient être inclus dans cette négociation, tels

(15) Ces principes concernent des contrats de travail à la fois souples et fiables, des politiques actives du marché du travail, des systèmes de formation au cours de la vie professionnelle, une sécurité sociale moderne. En tant que processus, ils préconisent le dialogue social. Une résolution du Conseil européen de décembre 2007 est venue ajouter d'autres principes aux quatre précédemment cités: flexibilité interne, modèles variés de flexicurité, solutions financières durables et prise en compte du genre. les congés parentaux ou congés de formation, qui apportent aussi flexibilité et sécurité. D'autre part, une dimension importante de la flexicurité concerne également les ajustements internes, ajustements au cycle économique et aux changements structurels sans licenciements par la ré-allocation interne des travailleurs, le changement des heures de travail, de l'organisation de travail, etc.

Mais les dispositions liées à la flexicurité, impliquant dans certains cas de passer d'un système (trop strict) de protection de l'emploi, au niveau de l'entreprise, à plus de protection sociale au niveau sociétal (16), peuvent-elles avoir pour résultat des réglages institutionnels «optimums»? À nouveau, la grande diversité des dispositions institutionnelles à travers le monde contribue à rendre malaisée toute réponse simple et directe et suggère la coexistence de différents modèles et non pas d'un modèle de flexicurité qui serait la panacée, soit un modèle de dimension unique convenant à tous les cas. Certains des modèles semblent produire des résultats supérieurs lorsqu'il est tenu compte à la fois des principes d'efficacité et d'équité.

Il existe cependant certains principes de base comme : 1) une législation fiable de la protection de l'emploi (LPE) et des conventions collectives sur la sécurité de l'emploi, qui cependant permet des ajustements de main-d'œuvre lorsque la nécessité économique s'en fait sentir ; 2) une protection sociale telle que les prestations d'assurance-chômage ou les politiques actives du marché du travail; 3) une série de «nouveaux» droits sociaux, tel le droit à la formation, le congé parental, les systèmes réversibles «temps partiel/temps plein», etc.; 4) un dialogue social bipartite et tripartite, ce dernier étant un élément obligatoire d'une flexicurité negociée. $\mathrm{Ne}$ pas tenir compte de cette quatrième condition peut entraîner un changement perçu comme étant imposé et/ou aboutir à des résultats sous-optimum, les partenaires sociaux véhiculant en ce sens informations et connaissances; d'autre part, l'inclusion des opinions des partenaires sociaux dans toute modification de réglementation concernant les droits du travail est une condition nécessaire (mais non suffisante) au sens de la politique économique des réformes du marché du travail. L'expérience faite en France dans le cadre du contrat de première embauche (CPE) et la décision, par les partenaires de la coalition en Allemagne, d'assortir les contrats de travail d'une période d'essai de deux ans, en sont des exemples. Tandis que dans le premier cas, la quatrième condition n'a pas été respectée, ce qui

(16) L'optimisation implique également, dans certains cas, un resserrement ou, du moins, une meilleure mise en oeuvre de la protection de l'emploi au niveau de l'entreprise, ainsi que la création d'un système de protection sociale plus adapté et plus généreux, offrant de bonnes garanties. Concernant les réformes du marché du travail, l'optimisation doit passer par le dialogue social. 
Figure 1 : De l'emploi à la sécurité du marché du travail

\author{
De la protection du poste de travail à la protection sur le marché du travail
}

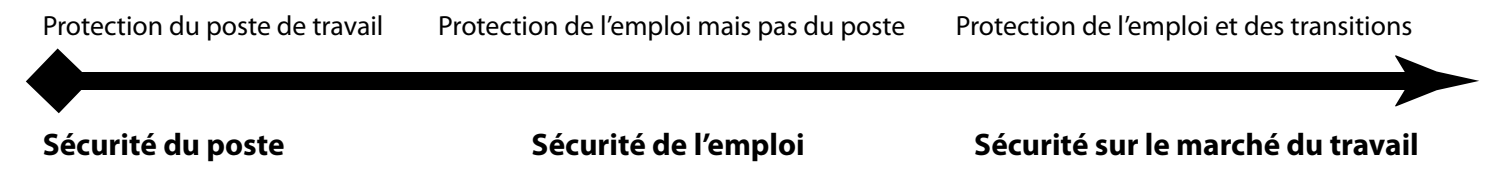

Source: adaptée d'Auer, 2008.

a finalement abouti à l'abolition du CPE et même du contrat nouvelle embauche (CNE), les syndicats ouvriers et le patronat, dans le second cas, se plaignent que la modification prévue (mais non mise en place) soit un pas menant à une détérioration plutôt qu'à une amélioration des conditions de travail et conditions économiques, ce qui montre que leurs informations et connaissances n'ont pas été pris en compte(17). Dans des environnements où ne règne qu'une faible confiance, la séquence des réformes, telle l'introduction d'éléments de sécurité crédibles avant celle d'éléments de flexibilité semble être cruciale. Le CPE a ainsi manqué son objectif et l'introduction tardive d'éléments de sécurité n'a pas suffi à convaincre les opposants.

En conclusion, il semble exister des combinaisons optimales entre protection de l'emploi et institutions et politiques du marché du travail, spécifiques à certains pays, apportant une protection tant sur le plan des revenus qu'au niveau des chances d'intégration sur le marché du travail et cette combinaison semble conférer plus de sécurité dans le marché du travail que les emplois stables seuls, dans un contexte d'incertitude. Ceci peut en partie également expliquer pourquoi au sein de pays affichant une telle combinaison, la qualité de l'emploi est plus élevée.

\section{Sécurité de l'emploi ou sécurité du marché du travail?}

La figure 1 illustre la modification et la transition s'opérant le long d'un axe temporel(18).

(17) Les syndicats font entendre leur plainte concernant la perte liée à la sécurité, qui affecte tant les salariés que les employeurs, et soulignent le fait que la présente législation, prenant en compte les contrats temporaires, vaut mieux qu'une réglementation qui ne tiendrait compte que des contrats à durée indéterminée assortis d'une période d'essai de deux ans.

(18) Tous les systèmes représentés sur cet axe temporel correspondent à des configurations à la fois spécifiques du système d'éducation et de formation et de l'organisation industrielle et du travail.
Alors que la Commission européenne considère la flexicurité comme un système de marché $\mathrm{du}$ travail marquant une transition entre sécurité du poste occupé (Job security: donc une sécurité de l'emploi sur un seul ou un éventail très restreint des postes en une seule entreprise) et sécurité de l'emploi (Employment security: sécurité de l'emploi assuré par plusieurs entreprises), le graphique montre qu'en réalité cette transition s'opère entre sécurité de l'emploi et sécurité du marché du travail. En effet, la transition entre sécurité du poste occupé et sécurité de l'emploi s'est opérée dans les années 1980 (selon une ampleur différente dans chaque pays), marquant la fin de la Job security qui était une protection très forte et assez rigide relative aux nécessités du changement technologique et organisationnel et englobait sécurité interne et externe. En effet, il était très fréquent, jusque dans les années 1980, que les travailleurs soient protégés au niveau d'une classification et d'une seule tâche, la protection s'appliquant à un poste bien défini. Les classifications des emplois et descriptions de poste étaient très strictes et changer de poste, même dans le cadre d'un contrat de travail maintenu, impliquait de négocier avec les syndicats. Au cours des années 1980, une transition s'est opérée vers des systèmes plus flexibles en interne, et passer d'un poste à un autre devint alors bien plus aisé. Mais, la flexibilité interne fut «troquée» au profit de la stabilité externe. Le prototype de ce système fut précisément le Japon qui a (toujours) un marché de travail interne flexible, mais les employeurs japonais garantissent en général des anciennetés longues à leurs salariés (19).

Le passage entre la Job security et Employment security s'est donc opéré avant la présente transition, qui ne se caractérise pas seulement par le fait que la protection de l'emploi est désormais garantie par plusieurs entreprises, mais plutôt par le fait que les périodes intermittentes sont (ou devraient être) protégées par de bonnes politiques de transition.

(19) Ce système ne s'applique en général qu'aux travailleurs réguliers, même si les travailleurs flexibles ont aussi des anciennetés plus longues (PASSET, 2003). 
Aujourd'hui, une bonne sécurité, non de l'emploi, mais du marché du travail, naît en règle générale pour le salarié de la combinaison des phases de protection de l'emploi dans une ou plusieurs entreprises et des phases intermittentes de protection assurées par des droits sociaux et des politiques du marché du travail effectives. Ce qui est garanti n'est pas la sécurité de l'emploi, mais plutôt l'employabilité et la sécurité des revenus. La sécurité englobe le système de prestations d'assurance-chômage, les politiques actives du marché du travail, les droits à la formation de même que les droits au congé de maternité et droits aux congés parentaux.

Dans certains de ces systèmes, une sécurité du marché du travail semble donc émerger: si la protection de l'emploi existe à la base, comme noyau central, elle est complétée par des mesures de sécurité plus larges que ce qu'une seule entreprise pourrait offrir. Mais il n'existe aucun système de sécurité du marché du travail qui soit de dimension unique et convienne à tous les cas. La sécurité du marché de travail peut être compatible avec différents degrés de protection de l'emploi, ainsi que des politiques du marché du travail différentes. Mais elle repose toujours à la fois sur la protection de l'emploi et sur la protection sociale active. Il existe donc des arguments forts en faveur de la "sécurité du marché du travail» (ou de la flexicurité) et des emplois flexibles, ces derniers étant intégrés à un système de protection sociale. La flexibilité, la stabilité et la sécurité peuvent se concevoir de manière dynamique en étant réparties sur l'ensemble du cycle de vie professionnelle des individus, avec des emplois flexibles lorsque les salariés démarrent dans la vie active, puis des emplois stables lorsqu'une famille se constitue, sans oublier les phases intermittentes de transition protégée (congé parental, périodes de formation, périodes brèves de chômage a taux de remplacement salarial relativement généreux, complétées par des programmes actifs du marché du travail, etc.). Mais pour être efficace, la sécurité du marché du travail a également besoin de zones stables d'emploi, à la fois dans le secteur public et le secteur privé. De fait, les transitions peuvent être tenues pour des passerelles menant à l'emploi stable et ne doivent pas être tenues pour des pièges générateurs d'exclusion.

\section{La dimension politico-économique de la flexicurité}

Dès avant la crise s'est dessinée autour de la flexicurité(20) une dimension politico-économique: il est évident que des formes concrètes de flexicurité

(20) La dimension politico-économique de la réforme du marché du travail se définit comme «la manière dont les facteurs politiques et institutionnels influencent l'élaboration, le processus décisionnel, l'adoption et l'application de la réforme» (OCDE, 2008). ne pourront découler que de négociations. Pour ceux qui s'y refuseront, c'est une situation d'impasse qui se dessinera: moins l'on négocie sur ces vastes questions, portant aussi bien sur la forme du contrat que sur la forme que doit revêtir le système de sécurité sociale, moins il sera possible d'obtenir des résultats en termes de réformes du marché du travail.

Que des éléments de ces systèmes soient ou non transposables est un point soumis à débat. (AlgAN, CAHUC (2006) avancent l'hypothèse que la flexicurité (en tant que système) ne peut être appliquée dans des environnements où ne règne qu'une faible confiance, par exemple la France. Mais les négociations récemment menées sur le sujet de la modernisation du marché du travail montrent qu'au moins certains des syndicats (parmi lesquels la CFDT et FO) s'impliquent dans des négociations ayant pour point central le thème de la flexicurité. Si une seule convention collective ne peut permettre de créer la version française de ce que doit être la flexicurité, des négociations répétées pourraient apporter certains changements, éventuellement même au régime français des relations du travail, connu pour être conflictuel.

Reste à savoir ce qu'il adviendra si la flexicurité échoue. Va-t-on vers une aggravation de la conflictualité qui sous-tend le compromis entre flexibilité et sécurité avec des syndicats exigeant la sécurité et des employeurs la flexibilité ? Ou assisterons-nous à la naissance d'un autre système de négociation sur les réformes du marché de travail? Si l'économie politique relative à la flexicurité se solde par une impasse, une modification du terme en «sécurité du marché du travail» serait alors judicieuse et opportune.

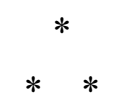

La mondialisation, bien qu'elle n'ait pas (encore?) mis fin aux contrats de travail de longue durée, accroît la flexibilité réelle et augmente la peur liée aux pertes d'emplois, amenant les salariés à accepter des conditions de travail moins favorables, tout en suscitant des craintes d'une détérioration constante des conditions de travail et de vie.

Des emplois qualitatifs et un travail décent, mais aussi des entreprises performantes nécessitent une stabilité structurelle du système de l'emploi, un environnement institutionnel sain, qui comprend des politiques du marché du travail fournissant un cadre d'ajustement où une sécurité transitionnelle est assurée. La mondialisation aura pour effet d'accroître le besoin d'assurance, face aux risques du marché du travail, ainsi que le besoin de transitions 
protégées, plutôt que d'atténuer celui-ci (AgelL, 1999; AUER et al., 2005).

Ce besoin de sécurité n'a plus à être défendu aujourd'hui dans un temps de crise financière qui a déjà atteint l'économie réelle. Il n'est pas clair encore si les systèmes qui ont opté pour une sécurité du marché de travail (ou flexicurité) tireront mieux leurs épingles du feu que les autres, de types flexibles à l'anglo-saxonne ou plus rigides comme ceux de l'Europe continentale ou encore du Sud. Un tout premier regard nous montre que dans une première phase (novembre 2007-novembre2008) les taux de chômage du regroupement continental ( $c f$. figure 5) semblent mieux résister (avec une diminution du taux de chômage de $-0,9$ point, principalement du à l'Allemagne ou le taux ne commence à remonter qu'à partir de décembre 2008) que les pays de «flexicurité» (augmentation de 1,2 point) ou encore de «flexibilité» (augmentation de 4,2 points dus principalement aux mauvais résultats de l'Irlande). Viennent ensuite les pays méditerranéens avec les plus mauvais résultats et un accroissement de 6,2 points surtout à cause des mauvais chiffres de l'Espagne.

Il est certainement trop tôt pour juger du rôle et de l'efficacité des institutions du marché de travail dans la gestion de la crise: le déclin du chômage en Allemagne semble principalement du à l'envolée des marchés mondiaux depuis 2005 et la chute peut être brutale dans l'avenir à cause de l'inversion de cette tendance. Quelques observateurs attribuent la diminution du chômage à l'effet des réformes Hartz sur le marché du travail, mais d'autres leur trouvent seulement un rôle marginal. En France, le chômage continue à monter depuis plusieurs mois. Le chômage partiel permet dans les deux pays d'atténuer la crise. Ces mesures d'ajustements internes sont d'ailleurs un élément de flexibilité d'ajustement et de sécurité des travailleurs sous-estimé dans ce pays souvent décrits comme rigides.

$\mathrm{Au}$ Danemark, le chômage a déjà commencé à augmenter, mais le taux de chômage reste, avec $4,1 \%$ en novembre 2008 , nettement en dessous des autres pays et le place en troisième position parmi les vingt-sept pays membres. Il est clair qu'un système de flexicurité comme au Danemark se voit confronté à des taux montants, car l'ajustement se fait de préférence externe sans trop d'utilisation des mesures internes. Les Pays-Bas, autre pays typique de «flexicurité» n'ont pas encore vue une augmentation des taux dans ce laps de temps, ce qui peut aussi être du à l'effet immédiat de la protection de l'emploi, plus stricte qu'au Danemark, ce qui a pour effet de maintenir l'emploi dans un premier temps. C'est finalement la durée de la crise qui dira si l'emploi peut être maintenu par des mesures internes ou s'il vaut mieux miser sur des institutions efficaces et protectrices d'ajustement externes. Car si les mesures internes touchent à leur fin, des licenciements s'en suivent et c'est finalement le système d'indemnité en place qui décidera sur la sécurité des travailleurs. Les travailleurs les plus vulnérables sont ceux des systèmes flexibles à l'anglo-saxonne, avec une montée rapide de chômage et une protection des chômeurs inadéquate. Aussi les pays plus rigides de l'Europe du Sud avec une protection de l'emploi forte, mais des marchés de travail segmentés et une protection contre le chômage tout aussi inadéquate sont peu à même d'assurer une bonne sécurité. Ainsi, l'Allemagne, après les réformes Hartz ne se trouve plus dans le peloton des pays qui assurent une bonne protection de revenu en temps de chômage, du moins pas pour ceux qui ne sont pas éligibles à la prestation de chômage après perte d'un emploi régulier.

La crise apportera donc des éléments de réponse sur la performance comparée des systèmes de protection sociale, en mettant à rude épreuve les systèmes de «sécurité du marché de travail» (ou de «flexicurité») jusqu'alors considérés comme des solutions d'agencement négocié «optimales» des institutions du marché du travail, liant des possibilités d'ajustement des entreprises avec une protection active des salariés et montrant une performance économique exceptionnelle du marché du travail et de la protection sociale. 


\section{Bibliographie}

Abraham K.G., Houseman S.N. (1995), "Labour Adjustment under Different Institutional Structures: A case study of Germany and the United States" in F. Buttler: Institutional Framework and Labour Market Performance: Comparative Views on the US and German Economies, Routledge, London.

Abraham K.G., Houseman S.N. (1994), “Does Employment Protection Inhibit Labor Market Flexibility: Lessons from Germany, France, and Belgium" in R. Blank (ed): Protection Versus Economic Flexibility: Is there a trade-off?, University of Chicago press.

Agell J. (1999), "On the benefits from rigid labor markets : norms, market failures, and social insurance", Economic Journal, Vol. 109.

Algan Y., CAhuc P. (2006), Civic attitudes and the design of labour market institutions: which countries can implement the Danish flexicurity model? CEPR-Discussion Paper Series No 5489.

AuER P. (2008), «La sécurité du marché du travail: comment conjuguer flexibilité et sécurité pour l'emploi décent», BIT, Cahiers de l'Économie et du marché du travail, 2008/2.

Auer P., Berg J., Coulibaly I. (2005), "Is a stable workforce good for the economy? Insights into the tenureproductivity-employment relationship", International Labour Review, Geneva, Vol. 144, Nr. 3.

Auer P., Efendioglu U., LeschKe J. (2005), Active Labour Market Policies around the World: Coping with the consequences of Globalisation, ILO, Geneva.

Auer P., Gazier B. (2006), L'introuvable sécurité de l'emploi, Paris, Flammarion.

Baker D., Glyn A., Howell D.R., Schmitt J. (2004), "Labor Market Institutions and Unemployment: A Critical Assessment of the Cross-Country Evidence" in: D.R. Howell (ed): Fighting Unemployment: The Limits of Free Market Orthodoxy, Oxford University Press.

BECK U. (2000), Die Zukunft von Arbeit und Demokratie, Frankfurt.

Betcherman G., Olivas K., Dar A. (2004), Impacts of active labor market programs: New evidence from evaluations with particular attention to developing and transition countries, Social Protection Discussion Paper Series 402, The World Bank, Washington, DC.

Blanchard O. (2005), "Designing Labor Market Institutions" in J. Restrepo and A. Tokman, (eds): Labor Markets and Institutions, Central Bank of Chile, Santiago.

Blanchard O., Tirole J. (2004), The optimal design of labor market institutions MIT, mimeo.

Blank R., Freeman R. (1994), "Does a larger Social safety net mean less Economic Flexibility" in R. Blank and R. Freeman (eds): Working under different rules. Russel Sage, New York.

Boeri T. (2005), Reforming Labor and Product Mar- kets: Some lessons from two decades of experiments in Europe: IMF working paper WP/05/97, Washington.

Bosch, G. (2003), Towards a new standard employment relationship in Western Europe? Manuscript presented at the $13^{\text {th }}$ World Congress of the International Industrial Relations Association, Berlin.

CAhuc P., Kramarz F. (2005), De la précarité à la mobilité: vers une sécurité sociale professionnelle, rapport au ministre d'État, ministre des Finances et de l'Industrie, et au ministre de l'Emploi du Travail et de la Cohesion sociale, La Documentation française.

Capelli P., Neumark D. (2004), "External Churning and Internal Flexibility: Evidence on the Functional Flexibility and Core-Periphery Hypotheses", Industrial Relations 43, 1, pp. 148-182.

Chatani Kazutoshi (forthcoming) "Flexicurity in Japan”, ILO, Geneva.

Clarc A., Postel-Vinay F. (2005), Job Security and Job Protection, CEP Discussion Papers DP678, Centre for Economic Performance, LSE (London School of Economics), updated version 2006.

Doogan K. (2002), Job insecurity and long-term employment in Europe, Paper presented at the Third International Congress of the Work and Labour Network, Osnabrück, 22-25 May.

Erlinghagen M., Knuth M. (2002), "Kein Turbo Arbeitsmarkt in Sicht: Fluktion stagniert, Beschäftigungsstabilität nimmt zu", IAT-report 4, Gelsenkirchen.

Elmeskov J., Martin J.P., Scarpetta St. (1998), "Key Lessons for Labour Market Reforms: Evidence from OECD Countries' Experiences", Swedish Economic Policy Review, Vol. 5, pp. 205-252.

European Commission (2003), Employment in Europe.

European COMMISSION (2001), Employment in Europe.

Freeman R.B. (2000), Single Peaked vs. Diversified Capitalism: The Relation between Economic Institutions and Outcomes, NBER Working paper 7556, NBER, Cambridge, MA.

FreEMAN R.B. (1998), "War of the models : which labour market institutions for the $21^{\text {st }}$ century?", Labour Economics, Vol. 5.

GAZIER B. (2003), Tous sublimes: vers un nouveau plein emploi, Paris, Flammarion.

Hall P., Soskice D. (2001), Varieties of Capitalism: The Institutional Foundations of Comparative Advantage, Oxford University Press.

Heckman J., Pagés C. (2000), The cost of job security regulation: Evidence from Latin American countries, NBER Working paper 7773, NBER, Cambridge, MA.

Hohendanner Ch., Bellmann L. (2006), “Interne und externe Flexibilität in WSI Mitteilungen" 5/2006. 
Huff Stevens A. (2005), The more things change, the more they stay the same: Trends in long-term employment in the United States, 1969-2002, NBER Working Paper 11878, Cambridge, MA, December.

ILO (2004), World Employment Report, Geneva.

ILO (1999), Decent Work, Geneva.

IZA(Institute for the study of labor) (2005), IZA compact, reporting on two studies (Verick, S. Threshold effects of dismissal protection legislation in Germany; and Bauer Th., Bender, S. and Bonin, H. Dismissal protection and worker flows in small establishments), January.

KNuth M. (2004), "Flexibilisation of Employment in the Knowledge Economy: Empirical Reality, Thrilling Menace or Wishful Thinking?", The first global labor forum, IIRA $5^{\text {th }}$ Asian Regional Congress, Seoul.

Kramarz F., Roux S. (1999) Within-Firm Seniority Structure and Firm Performance, Centre for Economic Performance Discussion Paper 420.

Lopez-Claros A., Porter M.E., Schwab K. (eds) (2005), The Global Competitiveness Report 2005-2006, World Economic Forum Geneva, Palgrave Macmillan, New York.

MAdSEn Per K. (2003), "Flexicurity through labour market policies and institutions in Denmark" in Auer P. and Cazes S. (2003), Employment stability in an age of flexibility, ILO

Marinescu I., (2006), Shortening the Tenure Clock: The Impact of Strengthened UK Job Security Legislation Job Market Paper, (version January), NBER, Cambridge, MA.

Martin J.P., GrubB D. (2001), "What works and for whom; A review of OECD countries' experiences with active labour market policies", Swedish Economic Policy Review, Vol. 8, pp. 9-56

Michie J., Sheehan M. (2003), "Labour market deregulation, 'flexibility' and innovation," Cambridge Journal of Economics 27, pp. 123-143.

Neumark D. (ed.) (2000), On the job: Is long-term employment a thing of the past? Russel Sage Foundation, New York.
OECD (1999, 2004, 2006), Employment Outlook, Paris.

OECD (2001), Knowledge, work organisation and economic growth, Labour Market and Social Policy Occasional Paper No. 50, DEELSA/ELSA/WD 20013, Paris.

Ono H. (2006), Lifetime employment in Japan: Concepts and Measurements, Working Paper No. 624, Stockholm School of Economics, March.

Pacelli L., Rapiti F., Revelli R. (1998), “Employment and Mobility of workers in industries with different intensity of innovation: Evidence on Italy from a panel of workers and firms", Economics of Innovation and New Technology.

RIFkin J., HeILBRONER R.L. (1995), The end of work: The decline of the global labor force and the dawn of the post-market era, New York.

RODRIK D. (1999), Institutions for high quality growth: What they are and how to acquire them, Paper for the IMF conference on Second Generation reforms, IMF, Washington.

Schmid G. (2002), "Employment insurance in critical transitions during the life-course" in P. Auer and B. Gazier: The future of work, employment and social protection, ILO/IILS, Geneva.

Sousa-PozA, A. (2004), "Job Stability and Job Security in Switzerland in the 1990s", European Journal of Industrial Relations, Vol. 10, 1, pp. 31-49, March.

Supiot A. (ed.) (1999), Au-delà de l'emploi, Report for the European Commission, Brussels.

Williamson O.E. (1985), The Economic Institutions of Capitalism: Firms, Markets, Relational Contracting, Free Press, New York.

Wilthagen T. (1998), Flexicurity: A New Paradigm for Labour Market Policy Reform? Discussion paper FS II 98-02. Wissenschaftszentrum für Sozialforschung, Berlin.

World Commission on the Social Dimension of GlobaliSATION (2004), A fair globalisation: Creating opportunities for all, ILO, Geneva. 
FORMATION EMPLOI

$\mathrm{N}^{\circ} 105$

\section{Janvier-Mars 2009}

- POURQUOI LES TRAVAILLEURS PRÉCAIRES NE PARTICIPENT-ILS PAS À LA FORMATION PROFESSIONNELLE CONTINUE ?

Quels sont les déterminants de la (non) participation des travailleurs précaires à la formation continue?

Coralie Perez

- DISCRIMINATIONS DANS L'ACCÈS AU STAGE : DU RESSENTI DES ÉLÈVES À L'INTERVENTION DES ENSEIGNANTS

Certains élèves semblent subir des discriminations dès l'accès au stage en lycée professionnel.

Nicolas Farvaque

\section{LA PLACE DES DIPLÔMES DANS LA} CARRIÈRE DES OUVRIERS DE LA FILIÈRE AUTOMOBILE

Quelle est la place des diplômes dans la sélection des ouvriers et dans leur promotion?

Armelle Gorgeu et René Mathieu

\section{LES DISTORSIONS DIPLÔMES/} QUALIFICATIONS : L'EXEMPLE DES TECHNICIENS ET AGENTS DE MAÎTRISE DE TYPE INDUSTRIEL

Les mutations du travail industriel provoquent des décalages entre les profils des jeunes diplômés et les attentes des employeurs. Paul Santelmann

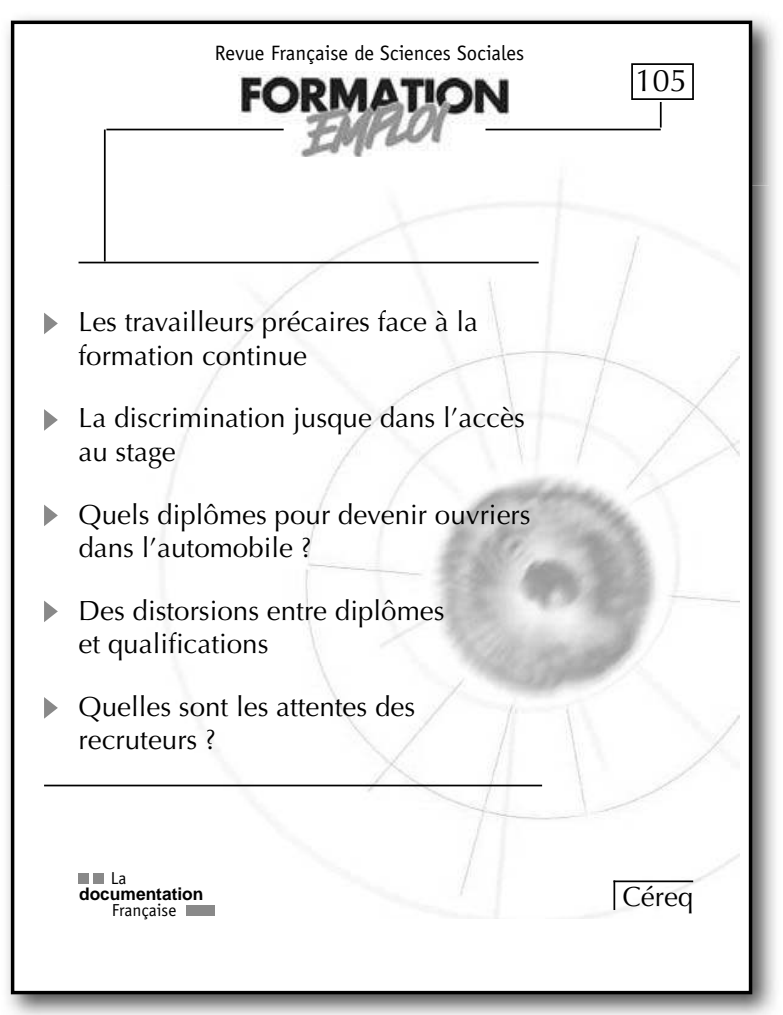

"QUI SE RESSEMBLE S'ASSEMBLE... » : LE RECRUTEMENT DES JEUNES DIPLÔMÉS DANS LES PROFESSIONS DE CADRE COMMERCIAL

Les recruteurs évaluent les candidats surtout à l'aune de leurs propres représentations. Oumaya Hidri

NOTE DE LECTURE

Présentation de l'ouvrage Mobilités étudiantes Sud Nord - Trajectoires scolaires de Marocains en France et d'insertion professionnelle au Maroc Par Catherine Agulhon

Le numéro : $19 € \bullet$ Le numéro spécial : $23 € \bullet$ L'abonnement un an (4 numéros) : France 57,50€ (TTC) • Europe $61 €$ $(\mathrm{TTC}) \bullet$ Dom/Tom 62,10€(HT) • Autres pays 65,70€(HT) • Supplément avion : 9,55€ • Commande adressée à : La Documentation française 124, rue Henri Barbusse 93308 Aubervilliers Cedex Tél. 0140157000 - Fax. 0140156800 